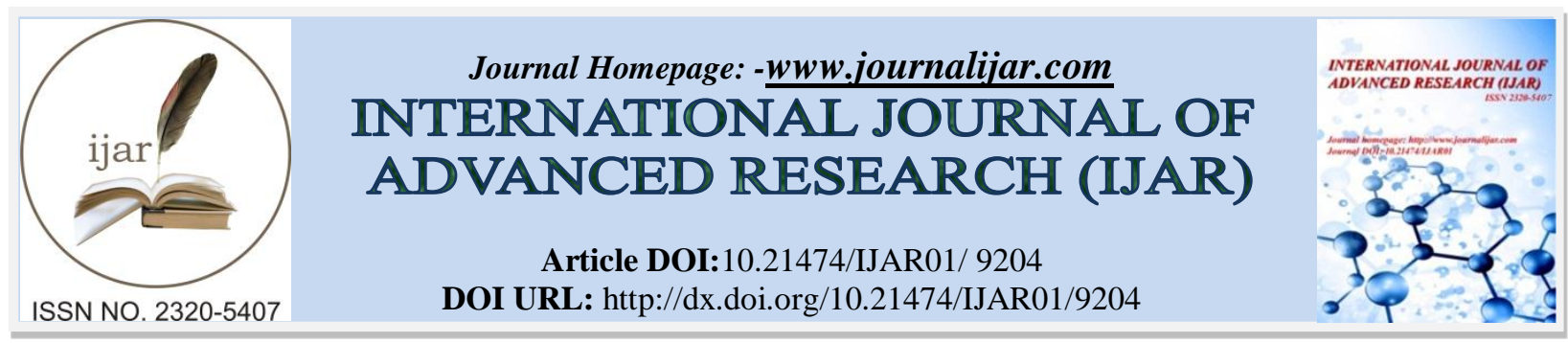

RESEARCH ARTICLE

\title{
EFFECTS OF FORWARD HEAD POSTURE ON POSTURAL BALANCE IN YOUNG ADULTS.
}

Dr. Darshana Jain ${ }^{1}$, Dr. Sukhada Prabhu ${ }^{2}$ and Dr. Manasi Desai ${ }^{3}$.

1. B.P.Th, D.Y. Patil University, School Of Physiotherapy, Nerul, Navi Mumbai, Maharashtra.

2. M.P.Th in Cardio Vascular and Respiratory Physiotherapy, Department Of Kinesiotherapeutics, Assistant Professor, D.Y. Patil University, School Of Physiotherapy, Nerul, Navi Mumbai, Maharashtra.

3. M.P.Th in Neurosciences, Department of Neuro Physiotherapy, Assistant Professor, D.Y. Patil University, School Of Physiotherapy, Nerul, Navi Mumbai, Maharashtra.

\section{Manuscript Info}

[........................

Manuscript History

Received: 04 April 2019

Final Accepted: 06 May 2019

Published: June 2019

Key words:-

Forward head posture, Balance,

Craniovertebral angle, Limits of

Stability, Balance master.

\section{Abstract}

Forward head Posture is nowadays one of the most common postural problems. Forward head posture can lead to upper cross syndrome, shortening of muscular fibers around articulation atlanto occipitals and overstretching of muscles around these joints. This can cause shift of line of gravity more anteriorly causing balance disturbances. Therefore this study aims to find the effect of forward head posture on postural balance in young adults.

Methodology: This study was conducted on 60 subjects which was divided into 2 groups i.e. control group individuals with the normal head posture \& individuals with the forward head posture. Assessment of forward head posture was done by taking the lateral view images of students and markings of the craniovertebral angle were done with the help of Kinovea software. Balance was assessed using Limits of stability component on Neurocom Balance master.

Result: In Limits of stability test for motor control ability assessment, the forward head group showed reduced motor control ability with significantly lower scores in all the five parameters. Forward head posture resulted in increase in reaction time, movement velocity, End point Excursion, Maximum excursion and Directional Control mainly in the Forward direction (left Forward, Right forward); Backward direction (Right back); left and Right directions respectively as per paired $t$ test. $(\mathrm{P}<0.05)$

Conclusion: From the above study we conclude that Individuals with forward head posture showed decreased balancing ability as compared with normal head posture group.

Copy Right, IJAR, 2019,. All rights reserved.

\section{Introduction:-}

Forward head is one of the leading postural problem. It is also called as Text Neck Syndrome, Wearsie Neck or Reading Neck. Head when positioned Anterior to the trunk, increasing the cervical convexity with the apex of lordotic cervical curve considering the distance from the line of gravity in optimal posture is known as Forward Head Posture. Recent Studies have shown that students are more prone to have Forward Head Posture due to 
excessive use of technology like smartphones, computers, excessive reading and writing in an abnormal posture. In modernized time, extended use of mobile phones and computers has increased the anterior weight bearing of cervical spine leading to variety of musculoskeletal disorders related to neck by changing biomechanical stress of cervical spine. Forward head posture is defined as any alignment in which external auditory meatus is positioned anteriorly to the plumb line through shoulder Joint. Forward head posture is generally recognized types of poor posture in sagittal plane. Head maintained forwardly for long periods of time causes Musculoskeletal disorders leading to reduced Lordosis of lower cervical in parallelism with kyphosis of upper thoracic vertebrae.

According to Panzer et al reported that abnormal posture, neck trauma, vestibular impairment contributed to abnormal biofeedback, function of maintaining balance. It might change torque required to maintain posture which is associated with reduced balance controlling ability. Hence it is important to evaluate the effect of postural changes in balancing abilities ${ }^{1}$. According to the Shivani Verma \& Jilani Shaikh in their research titled "Prevalence of Forward head posture among 12-16year old School going students" concluded that students are more prone to have forward head postures due to excessive use of technology like smartphones, computers, excessive reading and writing in an abnormal postures.This study identified the prevalence of FHP among school going students was $63 \%{ }^{2}$. According to Szeto et $\mathrm{al}^{3}$ and Moore stated that maintaining head posture for long periods of time causes musculoskeletal disorders leading to reduced Lordosis of the lower cervical in conjunction with kyphosis of the upper thoracic vertebrae ${ }^{4}$.

There is a recent trend to replace desktop with laptop computers. Most laptops are designed with screen joined to keyboard, making it possible to adjust separately in terms of screen height and distance, keyboard height and distance which leads to prolonged flexion at cervical spine with consequent higher activity in cervical erector spinae and upper trapezius muscles with posture in which trunk is slightly inclined backward. Burgess-limerick et al suggested that such posture causes shortening of muscular fibers around articulation atlanto-occipital and overstretching of muscles around joints and possibly chronic neck pain ${ }^{5}$. Silva et al reported in comparative study of people with or without non traumatic neck pain, that patients complaining of Non traumatic neck pain tend to keep forward head posture when compared to people without pain ${ }^{6}$.

Griegel-morris et al stated that forward head posture may affect not only neck but also thoracic spine and shoulder blade, possibly causing overall imbalance in musculoskeletal system ${ }^{7}$. Chester reported that severe neck pain was associated with decreased balancing ability ${ }^{8}$. Moreover Barrett et al stated that pain, inflammation or abnormal posture, reduced sense in joints and thereby abnormal proprioception which led to postural imbalance. However, there have been no studies directly evaluating only balancing ability, regarding neck, mainly in patients complaining of pain?

Maintaining upright body posture is fundamental for humans. Therefore, research concerning balance Control and Postural Stability are very diverse. Several Clinical and laboratory methods have been developed which enable researchers to assess different dimensions of the postural control system. Postural Balance refers to the ability to stay upright within base of support, or to recover equilibrium after external dynamic perturbation, whereas postural steadiness refers to standing as still as possible on a force plate. Limits of stability is amount of maximum excursion an individual is able to intentionally cover in any direction without losing the balance. Limit of stability measures postural instability while screening for individuals who are at higher risk of falling. Individuals with decreased Limit of stability have an increased risk of falling when they shift their body weight forward, backward or from side to side and hence are more prone to injuries. The parameters measured are reaction time in which time is taken by individual to start shifting his centre of gravity from static position after cue, measured in seconds; movement velocity which is average speed at which COG shifts; endpoint excursions is the distance willingly covered by subjects in first attempt towards target, maximum excursion is amount of distance actually covered by subject and directional control is comparison between amount of movement demonstrated in desired direction i.e. to amount of external movement in opposite direction of target expressed as percentage $\mathrm{e}^{\mathbf{1 0}}$

Therefore this study aims to find effect of forward head posture on postural balance in young students. To determine whether they have forward head posture with comparison to control group and then to evaluate balancing ability in students under assumption that they may show decreased body balancing ability even if they do not complain of pain. In the current study we are using Neurocom balance master which is the most reliable and valid assessment to measure the limits of balance stability. 


\section{Aim:}

To compare balance in Students with forward head posture and normal head posture.

\section{Objectives:}

1. To assess balance using limits of stability test on neurocom balance master in individuals with forward head posture.

2. To assess balance using limits of stability test on neurocom balance master in individuals with normal head posture.

3. To compare balance in students with forward head posture and normal head posture.

\section{Material and Methods:-}

Demographic details were collected via proforma. An ethical clearance was been obtained from the institution. This study was conducted on 60 subjects which was divided into 2 groups i.e. control group individual's with normal head posture \& individuals with forward head posture. Assessment of forward head posture is done by taking lateral view images of students and markings were done with help of Kinovea software. The intersection of a horizontal line passing through C7 spinous process and line joining the midpoint of tragus of ear is known as Craniovertebral angle. Smaller the craniovertebral angle more is the forward head posture. Craniovertebral angle $<53^{\circ}(\mathrm{FHP})$ and control group $>53^{0}$. Balance was assessed using Limits of stability component on Neurocom Balance master. Limit of stability for motor control ability evaluation is programme to assess subjects abilities to move their centre of gravity to fixed target points in eight directions: front, back, right, left, each diagonal direction with 5 parameters i.e. reaction time, movement velocity, directional control, endpoint excursions and maximum excursion.

Subjects were instructed to stand barefoot on force platform with their feet in a comfortable position, and their arms along their sides with palms directed towards the thighs. They were looking straight ahead with their head erect. The fixation point was placed $2 \mathrm{~m}$ away from the subjects on the wall in front of them. Subjects performed 10 consecutive trials of maximal voluntary forward leaning (limits of stability test - LOS test) with approximately 2 minutes rest between them. During the rest period subjects were asked to step off the platform and sit in order to avoid excessive fatigue. Each trial in the procedure started with 10 seconds of quiet standing, after which an acoustic signal was triggered to initiate the forward leaning phase. The subjects executed the leaning movement at their own pace until they reached their maximal range. Maximal leaning position achieved by the subjects was maintained until the end of the trial ( 15 seconds on average). Time of each trial was 30 seconds. During this time subjects were instructed not to raise their heels while performing the task. Otherwise the trial had to be repeated. Subjects were asked to execute the leaning phase mainly by changes in ankle joint angle.

The scores close to 100 represents no sway and reduced risk of fall, while scores close to 0 imply higher risk of falling.

\section{Statistical test:}

All statistical analysis were done using SPSS version 20.0 for windows. A p value of $<0.05$ was considered statistically significant using paired $\mathrm{T}$ tests. 


\section{Statistical Analysis:}

\section{Reaction Time}

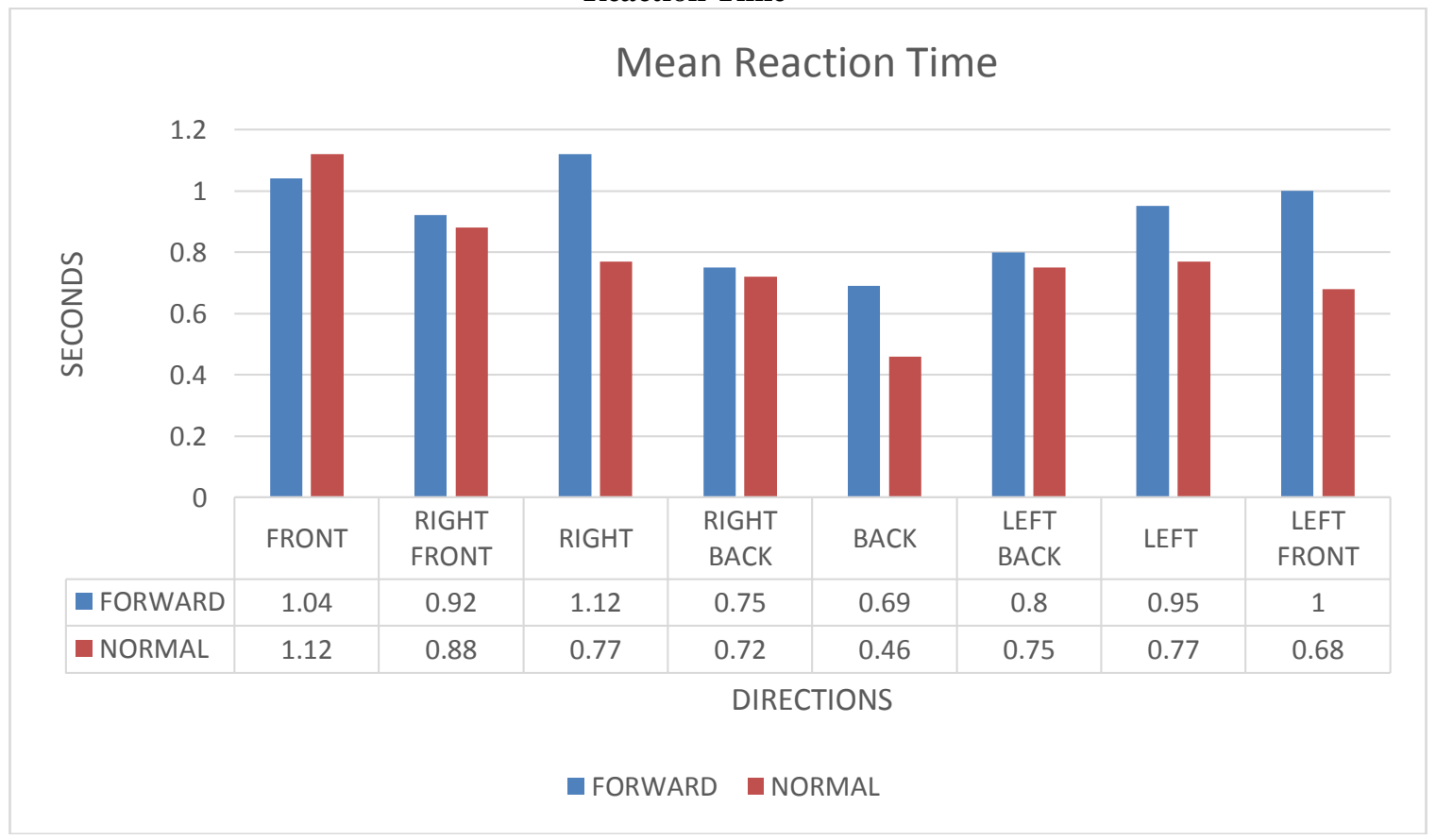

\begin{tabular}{|l|l|}
\hline DIRECTIONS & P VALUE \\
\hline FRONT & 0.528 \\
\hline RIGHT FRONT & 0.747 \\
\hline RIGHT & 0.012 \\
\hline RIGHT BACK & 0.796 \\
\hline BACK & 0.09 \\
\hline LEFT BACK & 0.620 \\
\hline LEFT & 0.167 \\
\hline LEFT FRONT & 0.072 \\
\hline
\end{tabular}

Table 1:-

Inference:

In Reaction Time, Right Direction (0.012) and Left Front Direction (0.072) are statistically significant with P value $<0.05$ 


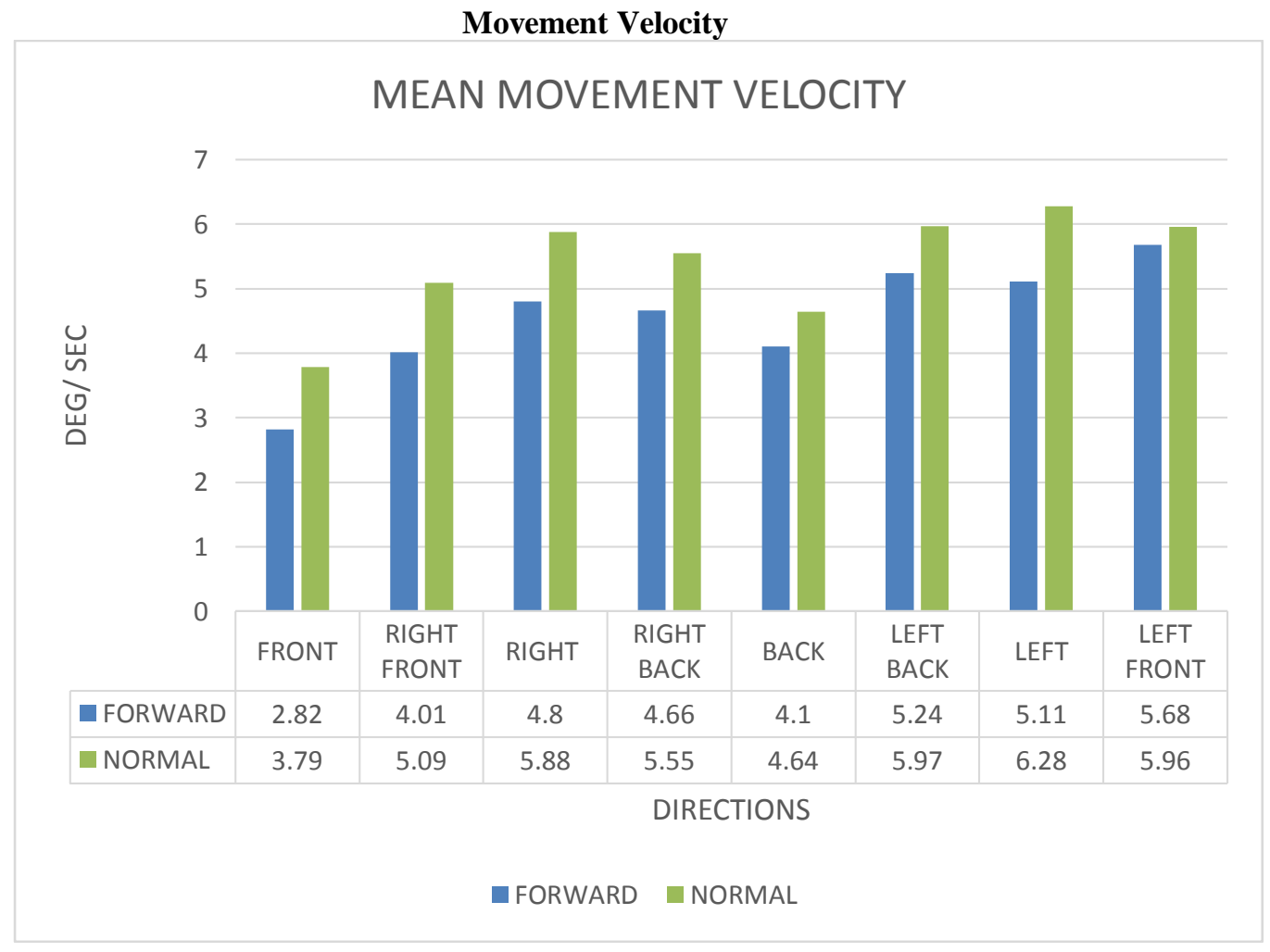

\begin{tabular}{|l|l|}
\hline DIRECTIONS & P VALUE \\
\hline FRONT & 0.25 \\
\hline RIGHT FRONT & 0.015 \\
\hline RIGHT & 0.091 \\
\hline RIGHT BACK & 0.044 \\
\hline BACK & 0.222 \\
\hline LEFT BACK & 0.192 \\
\hline LEFT & 0.038 \\
\hline LEFT FRONT & 0.831 \\
\hline
\end{tabular}

Table 2:-

Inference:

In Movement Velocity, Right front (0.015), Right (0.091), Right back (0.044), Left (0.038) directions are statistically significant with $\mathrm{P}$ value $<0.05$. 


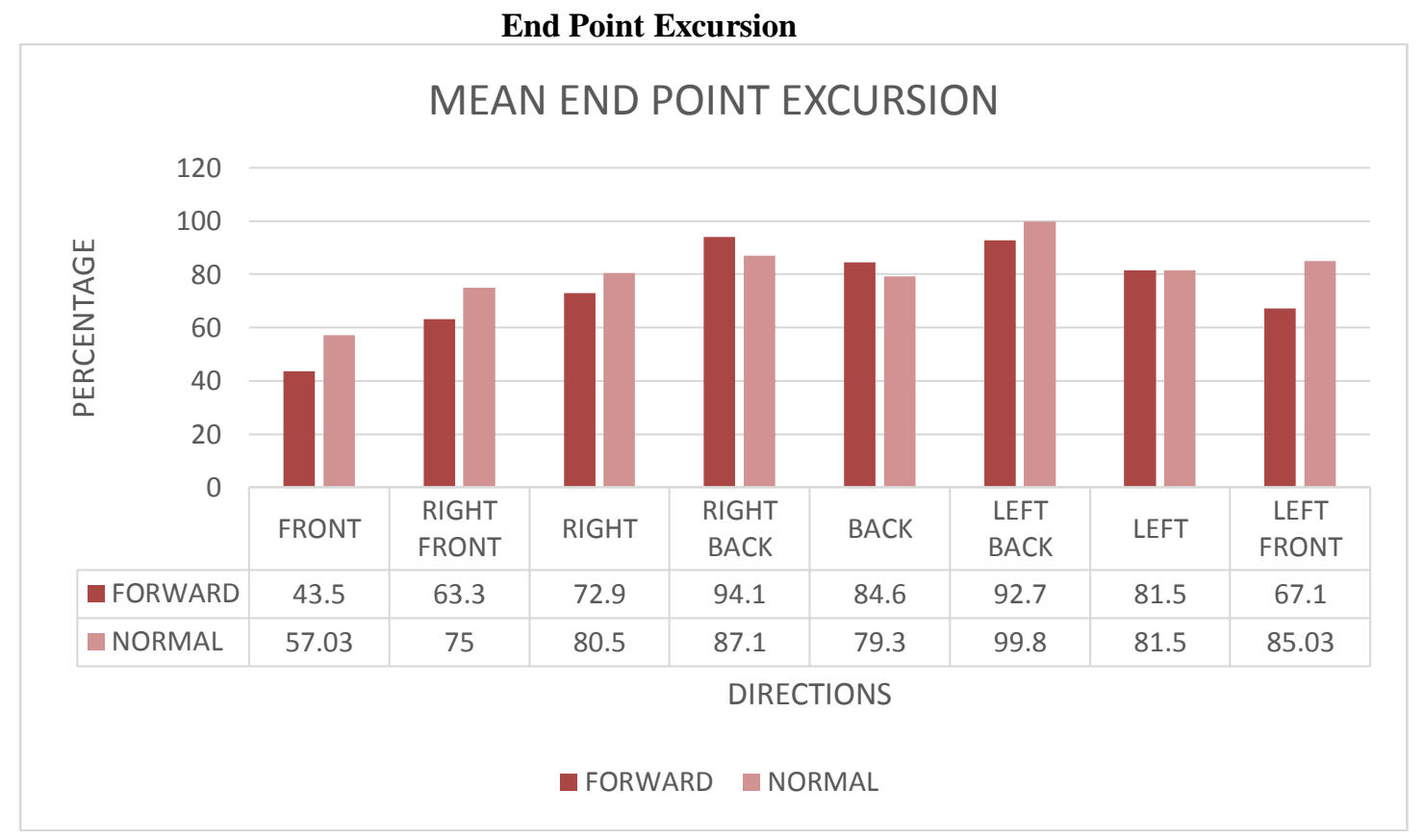

\begin{tabular}{|l|l|}
\hline DIRECTIONS & P VALUE \\
\hline FRONT & 0.06 \\
\hline RIGHT FRONT & 0.047 \\
\hline RIGHT & 0.175 \\
\hline RIGHT BACK & 0.399 \\
\hline BACK & 0.360 \\
\hline LEFT BACK & 0.333 \\
\hline LEFT & 0.003 \\
\hline LEFT FRONT & 0.003 \\
\hline
\end{tabular}

Table 3:- 


\section{Inference:}

In End point excursion, Right front (0.047), Left (0.003) and Left Front (0.003) is statistically Significant with P value $<0.05$.

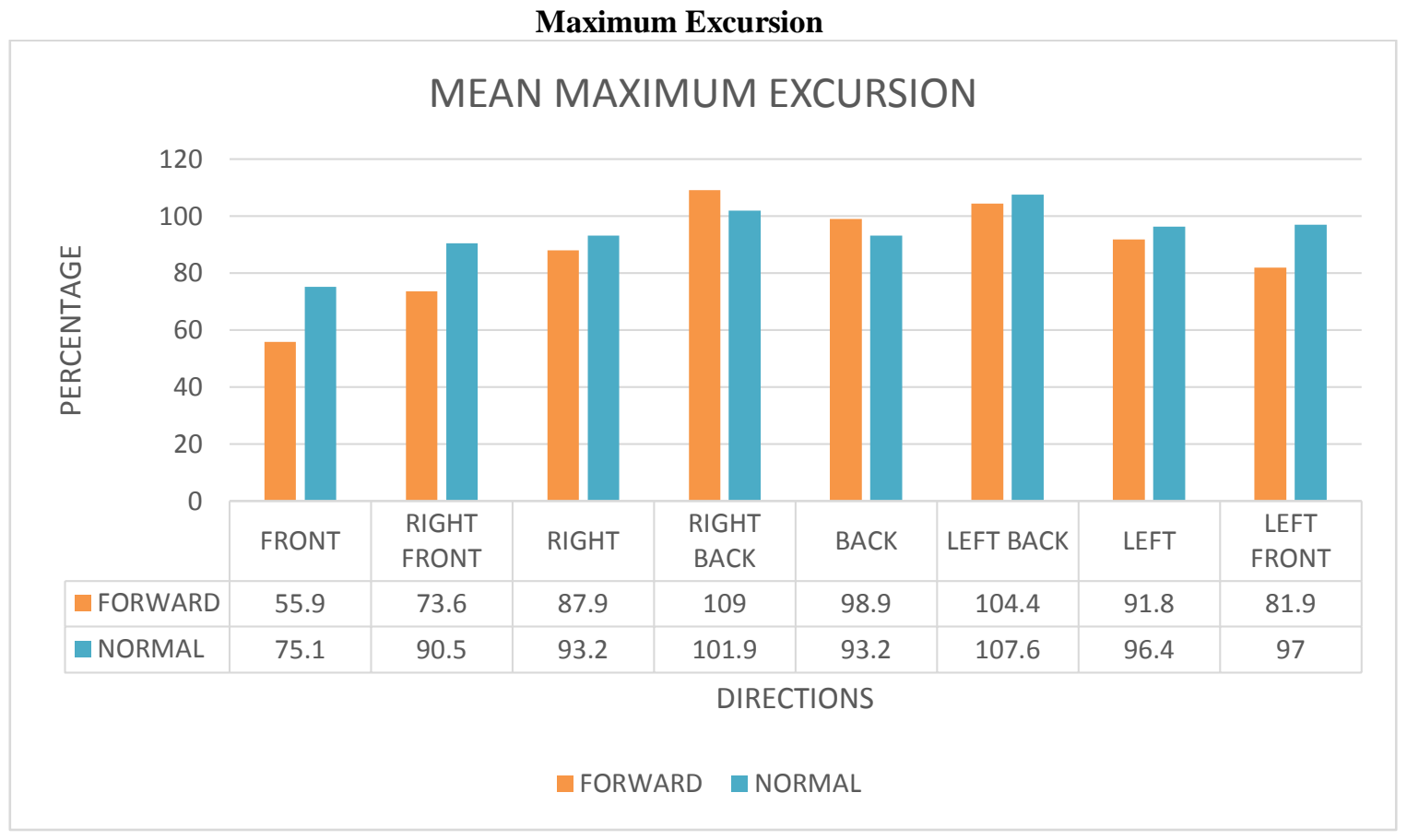

\begin{tabular}{|l|l|}
\hline DIRECTIONS & P VALUE \\
\hline FRONT & 0.001 \\
\hline RIGHT FRONT & 0.001 \\
\hline RIGHT & 0.162 \\
\hline RIGHT BACK & 0.038 \\
\hline BACK & 0.141 \\
\hline LEFT BACK & 0.354 \\
\hline LEFT & 0.097 \\
\hline LEFT FRONT & 0.003 \\
\hline
\end{tabular}

Table 4:-

\section{Inference:}

In Maximum excursion, Front (0.001), Right Front (0.001), Right back (0.038), Left (0.097) and Left Front (0.003) directions are statistically significant with $\mathrm{P}$ value $<0.05$ 


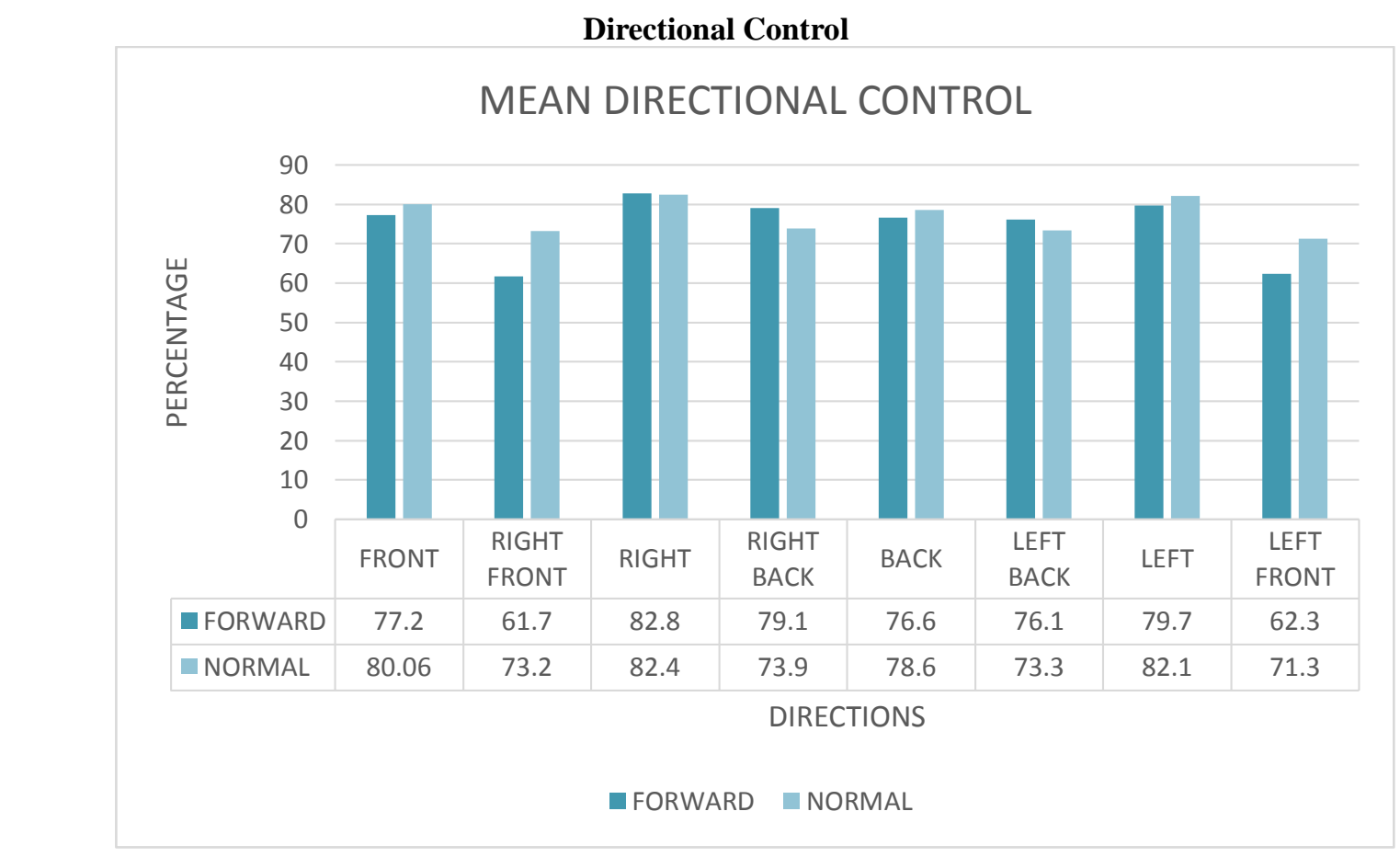

\begin{tabular}{|l|l|}
\hline DIRECTIONS & P VALUE \\
\hline FRONT & 0.461 \\
\hline RIGHT FRONT & 0.008 \\
\hline RIGHT & 0.828 \\
\hline RIGHT BACK & 0.154 \\
\hline BACK & 0.651 \\
\hline LEFT BACK & 0.522 \\
\hline LEFT & 0.202 \\
\hline LEFT FRONT & 0.031 \\
\hline
\end{tabular}

Table 5:-

\section{Inference:}

In Directional control, Right Front (0.008) and Left front (0.031) are statistically significant with P value $<0.05$

\section{Discussion:-}

Forward head posture is a common type of postural distortion seen in patients with neck disorders, there is change of head position that leads to changing of centre of gravity location ${ }^{15}$. Forward head posture is associated with many musculoskeletal problems ${ }^{\mathbf{1 6}}$, such as changing of mechanoreceptors, function, changing the sensitivity of neck muscles spindles ${ }^{17}$, coordination ${ }^{18}$ and affection balance ${ }^{19}$. The movement of head forward leads to increase the anterior curve in the lower cervical vertebrae and increase the posterior curve in the upper thoracic vertebrae to maintain balance, that causes musculoskeletal pains, visual disorders, headaches and other symptoms $\mathbf{2 0 - 2 2}^{\text {. }}$

There is a prevalence of forward head posture that may lead to chronic neck pain. The chronic neck pain may lead to affection of areas around the neck such as the thoracic vertebrae and shoulder, possibly causing an imbalance in the musculoskeletal system. Vuillerme N, Rougier P. in their research titled "Effects of head extension on undisturbed upright stance control in humans" reported that reduced sensation of joints is a major contributor to reducing balancing ability and have shown a deterioration of postural control when asking participants to stand with exaggerated extended head posture ${ }^{23-24}$. Due to technology advancement, students spend most of their time using cell phones, laptops etc. in abnormal head posture. 
Limits of stability are defined as the points at which the centre of gravity approach's the limits of the base of support and a correction strategy is required to return the centre of mass to within the base of support. It is a reliable variable of stability that provides information about voluntary motor control in the dynamic state. It helps to assess balance in dynamic state by instantaneously tracking the change in centre of Mass. A restricted limit of stability significantly influences the ability to react to perturbations in balance control system ${ }^{25}$.

And hence we undertook this study to find effect of forward head posture on postural balance in young students and to evaluate balancing ability in students with forward head and normal head posture under assumption that they may show decreased body balancing. The study was conducted and total 60 students were taken as subjects and were categorized into normal head and forward head posture according to kinovea software and all statistical analysis were done using SPSS version 20.0 for windows. A p value of $<0.05$ was considered statistically significant using $T$ tests.

Reaction Time can be defined as the time taken by an individual to start shifting his centre of gravity from the static position after the Cue or the onset of intentional movement toward the target as soon as the specific target appears on the screen, measured in Seconds. According to table 1, In mean Reaction Time, Right Direction (0.012) and Left Front Direction (0.072) are statistically significant with $\mathrm{P}$ value $<0.05$ due to the protruded head towards the anterior direction, the upper cervical vertebrae comes under extension moment. This causes a transition of centre of gravity to the anterior direction under both static and Dynamic conditions. As per our study, there were slight significant changes in reaction time in right and left front direction. In spite of exaggeratedly anterior location of COG with a protruded head, absence of body asymmetry in the right and left sides contribute to maintaining the ability to control direction and movement in the other direction. A delayed reaction time suggests that individual might have problems in cognitive processing ${ }^{26}$.

Movement velocity is the average speed of the center of gravity movement in degrees per second from the initial position to the target. As per table 2, in mean Movement Velocity, Right front (0.015), Right (0.091), Right back (0.044), Left (0.038) directions are statistically significant with $\mathrm{P}$ value $<0.05$ the reason attributed to this can be the forward COG that causes extended stretching of ankle joints to maintain body balance and the stretched ankle joints restrict movement of knee and hip joints. Therefore the forward head group has more restricted movement capacity of the lower limb joints than the normal head posture group, which makes it difficult to control velocity in anteroposterior body sway and also to lean the body to maximum extent while maintaining balance ${ }^{27}$.

End point Excursion is the distance of first movement towards the designated target and reflects the participant's perception of his own safety limits and is expressed as a percentage of Maximum LOS distance. Reduced movement velocities are indicative high-level of central nervous system deficits.

In table 3, End point excursion, Right front (0.047), Left (0.003) and Left Front (0.003) is statistically Significant with $\mathrm{P}$ value $<0.05$ In FHP there is increase extension of the atlanto-occipital joint and the upper cervical vertebrae as well as flexion of the lower cervical and upper thoracic vertebrae. Furthermore, this posture causes persistent and abnormal contraction of the suboccipital, neck, and shoulder muscles which causes a limitation to participation perception. In FHP, the centre of gravity (COG) of the head shifts in the anterosuperior direction, increasing the load on the neck, which causes dysfunction of the musculoskeletal, neuronal, and vascular systems ${ }^{28}$. Additionally, the muscles around the head and shoulders, including the trapezius, sternocleidomastoid, suboccipital, and temporal, are affected by FHP, which further worsens postural deformity. These changes cause persistent and abnormal pressure in the muscles, fascia, and nerves of the neck and shoulders, and rounding of the shoulders occurs to compensate for this deficit, which in turn, causes a high load on the superior trapezius and levator scapula muscles ${ }^{29}$. All of these changes eventually cause tension neck syndrome. Persistent tension in the head and posterior neck muscles can pathologically mimic tension headache. Additionally, FHP alters the COG of the body that lead to mechanical modifications related to postural control in the torso and every joint. The body attempts to adapt to these changes by altering its balance control mechanisms; these adaptations decrease balance ability while performing different activities and increase the risk of falling and musculoskeletal injury, and ultimately result in limited body function and a high incidence of various disease ${ }^{30}$.

According to table 4, Maximum Excursion is the amount of distance the subject actually covers or moved his COG. In Maximum excursion, Front (0.001), Right Front (0.001), Right back (0.038), Left (0.097) and Left Front (0.003) directions are statistically significant with $\mathrm{P}$ value $<0.05$. If the COG of the body shift from its normal Position, high 
muscle activity is needed to recover from this unstable position. Therefore in conditions of structural imbalance such as forward head posture, it is difficult to properly adjust changes in the external environment because the autonomic nervous system has decreased ability to respond to external stimulation ${ }^{31}$.

Directional Control is comparison between amount of movement demonstrated in the desired direction; i.e. towards the target to the amount of external movement. As per table 5 In Directional control, Right Front (0.008) and Left front $(0.031)$ are statistically significant with $\mathrm{P}$ value $<0.05$. The result of present study were in accordance to those reported by Johnson et al, who tried to find the effect of head position on postural control. They found head extension impacted postural control compared with head in neutral as indicated by increase COP velocity in the anterior/posterior direction and a reduction in the time to contact the anterior/posterior stability boundary. Kang et al. suggested that forward head posture maybe associated with changes in postural control. They found that young healthy computer workers had low balance scores and a more pronounced forward head posture. Reduced Endpoint excursions, excessively larger maximum excursions and poor directional control are all indicative of motor control abnormalities ${ }^{32}$.

In the Current study, LOS test for motor control ability assessment, the forward head group showed reduced motor control ability with significantly lower scores in reaction time, movement velocity, End point Excursion, Maximum excursion and Directional Control mainly in front directions.

\section{Conclusion:-}

In Limits of stability test for motor control ability assessment, the forward head group showed reduced motor control ability with significantly lower scores in all five parameters. Forward head posture resulted in increase in reaction time, movement velocity, End point Excursion, Maximum excursion and Directional Control mainly in Front direction (left front, Right front); Back direction (Right back); left and Right directions respectively. Forward head posture group showed decreased balancing ability as compared to normal head posture group as there is shift of gravity in anterior direction causing more balance disturbances.

1. In reaction time, Right Direction (0.012) and Left Front Direction (0.072) are statistically significant with P value $<0.05$

2. In Movement Velocity, Right front (0.015), Right (0.091), Right back (0.044), Left (0.038) directions are statistically significant with $\mathrm{P}$ value $<0.05$.

3. In End point excursion, Right front (0.047), Left (0.003) and Left Front (0.003) is statistically Significant with P value $<0.05$.

4. In Maximum excursion, Front (0.001), Right Front (0.001), Right back (0.038), Left (0.097) and Left Front (0.003) directions are statistically significant with $\mathrm{P}$ value $<0.05$.

5. In Directional control, Right Front (0.008) and Left front (0.031) are statistically significant with $\mathrm{P}$ value $<0.05$.

\section{Bibilography:-}

1. Drashti Talati, G. Varadhrajulu, Mandar Malwade. The Effect of Forward Head Posture On Spinal Curvatures in Healthy Subjects. Asian Pacific Journal Of Health Sciences; Volume 5; 2018.

2. Shivani Lalitkumar Verma, Jilani shaikh, Ranveer Mahato, Megha Sandeep Sheth. Prevalence Of Forward Head Posture among 12-16 year old school going students- A Cross sectional study. Applied Medical Research, 2018 Vol 4, No. 2, Page 18-21.

3. Szeto GP, Straker L, Raine S. A Field Comparison of neck and shoulder postures in symptomatic and asymptomatic office workers. Appl Ergon 2002; 33: 75-84

4. Moore MK, Upper Crossed Syndrome and its relationship to Cervicogenic headache. J Manipulative Physiol Ther 2004; 27: 414-420

5. Burgess-Limerick R, Plooy A, Ankrum DR. The effect of imposed and self-selected computer monitor height on posture and gaze angle. Clin Biomech (Bristol, Avon) 1998;13:584-592.

6. Silva AG, Punt TD, Sharples P, Vilas-Boas JP, Johnson MI. Head posture and neck pain of chronic nontraumatic origin: a comparison between patients and pain-free persons. Arch Phys Med Rehabil. 2009; 90:669-674.

7. Griegel-Morris P, Larson K, Mueller-Klaus K, Oatis CA. Incidence of common postural abnormalities in the cervical, shoulder and thoracic regions and their association with pain in two age groups of healthy subjects. Phys Ther. 1992; 72:425-431.

8. Chester JB., Jr Whiplash, postural control and the inner ear. Spine. 1991; 16:716-720. 
9. Barrett DS, Cobb AG, Bentley G. Joint proprioception in normal, osteoarthritic and replaced knees. J Bone Joint Surg Br. 1991; 73:53-56.

10. Grzegorz Juras, Kajetan Stomka, Artur Fredyk, Grzegorz Sobota, Bogdan Bacik. Evaluation Of the limits of stability balance test. Journal of human kinetics volume 19 2008, 39-52.

11. Joon-Hee Lee. "Effects of forward head posture on static and dynamic balance control. J Phys Ther Sci. 2016 Jan; 28 (1): 274-277

12. Kang JH, Park RY, Lee SJ, Kim JY, Yoon SR, Jung KI. "The effect of the forward head posture on postural balance in long time computer based worker. Ann Rehabil Med. 2012 Feb;36(1):98-104

13. Abdelrhman I Abdelghany, Maher Ahmed Elkablawy, Shaimaa El Gharib Ali Salem, and NagiZak Ahmed." Relationship between head postural changes and dynamic balance in a symptomatic forward head posture student. IJPRIF 2016, Vol.9, No.7, pp 93-98.

14. Asmita Karajgi, Anuprita Thakur, Cresida D'souza, Twinkle Dabholkar, Unnati Pandit and Sujata Yardi in their research titled "Effects of Forward Head Posture on Balance in Asymptomatic Young Adults. Indian Journal of Public Health Research and Development july 2015 6(3):123

15. Yip, Chris Ho Ting, Thomas Tai Wing Chiu, and Anthony Tung Kuen Poon. "The relationship between head posture and severity and disability of patients with neck pain." Manual therapy 13.2 (2008): 148- 154.

16. Haughie, Laura J., Ira M. Fiebert, and Kathryn E. Roach. "Relationship of forward head posture and cervical backward bending to neck pain." Journal of Manual \& Manipulative Therapy 3.3 (1995): 91- 97.

17. Lee, Mi-Young, Hae-Yong Lee, and Min-Sik Yong. "Characteristics of cervical position sense in subjects with forward head posture." Journal of physical therapy science 26.11 (2014): 1741-174.

18. Röijezon, Ulrik, et al. "A novel method for neck coordination exercise-a pilot study on persons with chronic non-specific neck pain." Journal of neuroengineering and rehabilitation 5.1 (2008): 1.

19. Michaelson, Peter, et al. "Vertical posture and head stability in patients with chronic neck pain." Journal of Rehabilitation Medicine 35.5 (2003): 229-235.

20. Cho WH, Lee WY, Choi HK. An investigation on the biomechanical effects of turtle neck syndrome through EMG analysis. J Korean Soc Precision Eng 2008; 1:195-196.

21. Rempel, David M., Robert J. Harrison, and Scott Barnhart. "Work-related cumulative trauma disorders of the upper extremity." Jama 267.6 (1992): 838-842.

22. Picavet, H. S. J., and J. S. A. G. Schouten. "Musculoskeletal pain in the Netherlands: prevalence, consequences and risk groups, the DMC 3-study." Pain 102.1 (2003): 167-178

23. Kogler A, LindforsJ, Odkvist LM, Ledin T. Postural stability using different neck positions in normal subjects and patients with neck trauma. ActaOtolaryn- gologica 2000; 120(2):151-5.

24. Vuillerme N, Rougier P. Effects of head extension on undisturbed upright stance control in humans. Gait and Posture 2005; 21(3):318-25.

25. Sean Clark, MS, Debra J Rose. Generalizability of the limits of stability test in the evaluation of dynamic balance among older adults. Arcs Phys Med Rehabil 1997; 78:1078-84

26. Di Fabio RP. Sensitivity and specificity of platform Posturography for identifying patients with vestibular dysfunction. Phys Ther 1995; 75: 290-305

27. Vernazza S, Alexandrov A, Massion J. is the center of gravity controlled during upper trunk movements. Neuroscience Lett 1996; 206: 77-80

28. Harrison DE, Harrison DD, Betz JJ, et al.: Increasing the cervical lordosis with Chiropractic biophysics seated combined extension-compression and transverse load cervical traction with cervical manipulation: nonrandomized clinical control trial. J Manipulative Physiol Ther, 2003, 26: 139-151.

29. Cole AK, McGrath ML, Harrington SE, et al.: Scapular bracing and alteration of posture and muscle activity in overhead athletes with poor posture. J Athl Train, 2013, 48: 12-24.

30. Lee CM, Jeong EH, Freivalds A: Biomechanical effects of wearing high-heeled shoes. Int J Ind Ergon, 2001, 28: 321-326.

31. Ki SH, Song YK: Correlations of forward head posture to heart rate variability and standing posture balance factors. J Korean Med Rehab, 2014, 24: 163-176

32. Johnson, Molly B and Richard EA Van Emmerik. "effect of head orientation on postural control during upright stance and forward lean.” Motor control 16.1 (2012): 81-93. 